\title{
Electret condenser microphone as a traditional Chinese medicine arterial pulse sensor
}

\author{
Erni Yudaningtyas ${ }^{1 *}$, Waru Djuriatno ${ }^{1}$, Achsanul Khabib ${ }^{2}$ and Dionysius J.D.H. Santjojo ${ }^{3}$ \\ ${ }^{1}$ University of Brawijaya, Department of Electrical Engineering, J1. M. T. Haryono 167, Malang, Indonesia \\ ${ }^{2}$ State Polytechnic of Malang, Department of Electrical Engineering, Jl. Soekarno - Hatta 9, Malang-Indonesia \\ ${ }^{3}$ University of Brawijaya, Department of Physics, J1. Veteran, Malang-Indonesia
}

\begin{abstract}
Arterial pulse signal reading by a therapist in Traditional Chinese Medicine (TCM) requires considerable experience and thoroughness. The interpretation resulted by the therapist is very subjective. The objectivity of the device can be achieved by increasing the accuracy and the consistency of the reading in the range of 1-2 Hz. An electret condenser microphone (ECM) can be used as a pulse sensor in such device. This study reveals that ECM performs a number of advantages such as small-sized and inexpensive. Unfortunately, this ECM also picks up mechanical noises during direct contact with the subject. This study proposed soft silicon rubber as a medium between subjects and ECM. The technique was designed to enhance the mechanical coupling between the ECM and the source signal without affecting the signal source. The results show that the ECM could measure the signal of $0.5-10 \mathrm{~Hz}$ with the average accuracy of $99,47 \%$.
\end{abstract}

\section{Introduction}

Arterial pulse readings by a therapist in Traditional Chinese Medicine requires years of experience to produce an accurate diagnosis. Today, many efforts have been made to make a diagnostic instrument functioning similar procedure in the absence of a professional therapist. The most important part of the instrument is the pulse sensor.

Researchers around the world have been proposed different kinds of sensor to detect the pulse such as piezoelectric devices [1], strain gauges [2], [3], condenser microphones [4], etc. In our previous work, a strain gauge model $318 \mathrm{~L} \mathrm{SS}$ model 86 as a heartbeat sensor. The sensor was used to capture the signal with the frequency range $0.26-1.51 \mathrm{~Hz}$ [5]. The strain gauge study was focused on signal processing analysis using FFT technique [6].

There was also a proposed technique utilizing a strain gauge beam array that can work simultaneously to capture Cun, Guan, and Chi signal which were processed by a computer software. All of the systems utilized sensor holders to easily fix positions during the measurements [7]. However, the size of the strain gauge was too large.

Another researcher proposed the use of a flexible piezoresistive pressure sensor in the form of radial pulse tonometry (RPT) sensor consisting of 6 piezo-resistive pressure sensors (C33 series, EPCOS, Germany). The piezo-resistive is designed with Wheat-Stone bridge with four active arms. The size of the sensor was $1.00 \mathrm{~mm} \times$ $1.00 \mathrm{~mm}$, and $\mathrm{mV}$ outputs were generated according to the applied pressure [8]. However, the accuracy of the measurement was not determined only by the distribution of the small sensor in the array related to the smallest point of the pulse should be measured. Departing from this problem another researcher proposed a more precise type of sensor utilized a capacitive sensor. This sensor was able to detect the pulse of each area in a dynamic and static range because the capacitive properties arranged in an array simultaneously samples the dynamic and static pressure at each area on the wrist pulse sensing area [9].

However, disputes were raised since the use of capacitive sensors array has not been acknowledged in the measurement standard. Standardization was then established from the use of this sensor namely the Simultaneously Palpation (SP) and Pressing with One Finger (PWOF) method [10]. Since the dimensions of the capacitive sensors arranged in this array have large dimensions, a smaller type of sensor, an electret condenser microphone (ECM) was proposed.

The ECM is a microphone that works in the range of 20-20.000 Hz. Some researchers have been proposing this ECM for the pulse sensor with the use of air medium [4]. The use of air as the medium in low-frequency range has a weakness which comes from energy transfer from the vibration source to the sensor. Energy transfer from the air also includes noise. This noise comes from the air vibration around the device or from the friction occurring during the measurement.

\footnotetext{
* Corresponding author: erni@ub.ac.id
} 


\section{Experimental setup}

The main principle of energy transfer when passing soft silicone rubber is better than passing through the air medium. This is because the medium propagation with soft silicone rubber particle density is better than using more tenuous particles. The weakness of the air medium also resulted in various noise will enter, among others, noise due to other sounds coming in or the noise that arises due to friction during measurement.

Some shortcomings of the above ECM can be reduced by designing filters. There are different kinds of filters that can be used. One of them is a mechanical filter. This filter can be used to reduce the interception of noise from outside. Furthermore, a soft silicone rubber can be used to increased the energy transfer and hence the signal to noise ratio.

The propagation of energy through the medium can be weakened, this is caused by various things such as distance and material from the inhibiting medium. This term is called acoustic attenuation, the effect of acoustic attenuation can affect the change in the output amplitude. This amplitude can be seen in the formulation below [11]:

$$
A=A_{0} e^{-a d}
$$

where $A_{0}$ is amplitude of the propagating wave at a given location, $A$ is reduced amplitude at another location, $d$ is distance travelled between the two locations and $a$ is attenuation coefficient in neper $(N p) /$ length (can be converted to $d B /$ length by dividing of 0.1151 ).

Acoustic impedance is assumed to be how a particle of material distributes a wave; it is closely related to the resistance of a material and the propagation wave that it carries [12]. It is calculated by the product of the material's density and it's longitudinal wave speed as shown in Equation below

$$
Z=\rho c
$$

where $Z$ is acoustic impedance (Rayl $=\mathrm{kg} / \mathrm{m}^{3} \cdot \mathrm{m}$ ) $s), \rho$ is density and $c$ is longitudinal wave speed. In this research, silicone rubber longitudinal wave speed is 0,94 $\mathrm{km} / \mathrm{s}$ and the acoustic impedance is 1,4 MRayl [13].

The noise filter serves as a mechanical filter for the noise that arises from the outside does not enter into the microphone which showed in Fig. 1 point 2. The microphone used is a type of ECM sold commercially. Before being used to measure pulse signals, this microphone must be tested whether the microphone can capture mechanical signals accurately. Signals are provided with varying ranges to know the working range of the microphone.

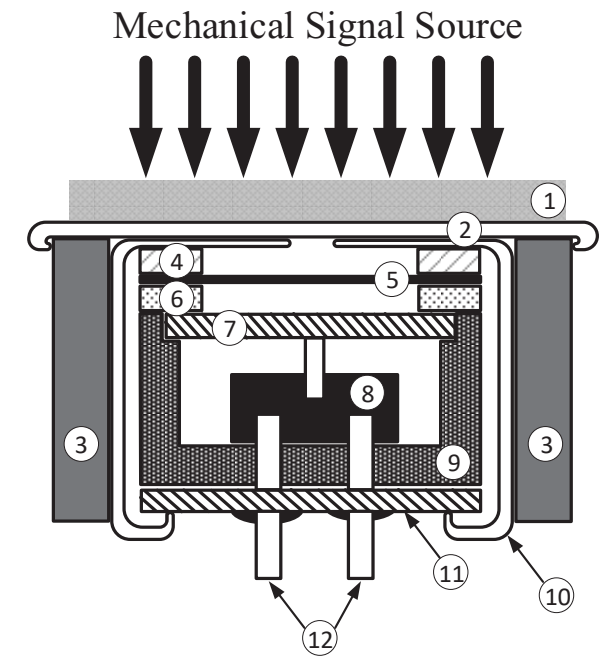

Fig. 1. Schematic diagram of the pulse sensor.

Fig. 1 shows the model of the schematic diagram of ECM consist of: (1) Soft silicone rubber, (2) Noise filter, (3) Rubber housing, (4) Metal Washer, (5) Electret Material, (6) Isolator Spacer, (7) Pick Up Plate, (8) Field Effect Transistor, (9) Housing of Field Effect Transistor, (10) Metal Shield, (11) PCB Isolator, (12) Terminal lead. The most important thing of this ECM design is the addition of soft filter material that serves as a mechanical filter. This filter can reduce the mechanical noise that occurs when the measurement takes place or noise can arise from the sounds that surround the measuring environment.

\begin{tabular}{|c|c|c|}
\hline Parameter & Conditions & Value \\
\hline sensitivity (S) & $\begin{array}{c}\mathrm{f}=1 \mathrm{kHz}, 1 \mathrm{~Pa}, 0 \\
\mathrm{~dB}=1 \mathrm{~V} / 1 \mathrm{~Pa}\end{array}$ & $-44 \mathrm{~dB}$ \\
\hline operating voltage & & $3-10 \mathrm{Vdc}$ \\
\hline $\begin{array}{l}\text { output impedance } \\
\text { (Zout) }\end{array}$ & $\mathrm{f}=1 \mathrm{kHz}, 1 \mathrm{~Pa}$ & $2.2 \mathrm{~K} \Omega$ \\
\hline $\begin{array}{l}\text { sensitivity reduction } \\
\left(\Delta \mathrm{S}-\mathrm{V}_{\mathrm{s}}\right)\end{array}$ & $\begin{aligned} \mathrm{f} & =1 \mathrm{kHz}, 1 \mathrm{~Pa}, \mathrm{Vs} \\
& =3.0 \text { to } 2.0 \mathrm{Vdc}\end{aligned}$ & $-3 \mathrm{~dB}$ \\
\hline frequency (f) & & $\begin{array}{c}20-20,000 \\
\mathrm{~Hz} \\
\end{array}$ \\
\hline $\begin{array}{l}\text { current consumption } \\
\text { (IDSS) }\end{array}$ & $\begin{array}{c}\mathrm{Vs}=3.0 \mathrm{Vdc}, \mathrm{RL}= \\
2.2 \mathrm{~K} \Omega\end{array}$ & $0.5 \mathrm{~mA}$ \\
\hline $\begin{array}{l}\text { signal to noise ratio } \\
(\mathrm{S} / \mathrm{N})\end{array}$ & $\begin{array}{c}\mathrm{f}=1 \mathrm{kHz}, 1 \mathrm{~Pa}, \mathrm{~A}- \\
\text { weighted }\end{array}$ & $60 \mathrm{dBA}$ \\
\hline operating temperature & & $-20-70^{\circ} \mathrm{C}$ \\
\hline Dimension & & $\begin{array}{c}\varnothing 9.7 \times 4.5 \\
\mathrm{~mm}\end{array}$ \\
\hline Weight & & $0.8 \mathrm{gr}$ \\
\hline Material & & $\mathrm{Al}$ \\
\hline
\end{tabular}

Table 1. The Specification of ECM [14].

Note: We use the "Pascal (Pa)" indication of sensitivity as per the recomendation of I.E.C. (International Electrotechnical Commission). The sensitivity of "Pa" will increase $20 \mathrm{~dB}$ compared to the " $\mu$ bar" indication. Example: $-60 \mathrm{~dB}(0 \mathrm{~dB}=1 \mathrm{~V} / \mu \mathrm{bar})=-40 \mathrm{~dB}(1 \mathrm{~V} / \mathrm{Pa})$

Table 1 shows the original specifications of ECM issued fabrication. From the table can be seen that the frequency range of the microphone is $20-20.000 \mathrm{~Hz}$. This microphone also has a small dimension and weights less than 1 gram. 


\section{Results and discussion}

To be able to know the characteristics and responses of the microphone, the authors tested the system using a sound source with a constant amplitude and a varying frequency that has a range of $0-10 \mathrm{~Hz}$. This was intended to capture the pulse signals that have variable frequency components. Experimental setup of this research can be seen in Fig. 2 below.

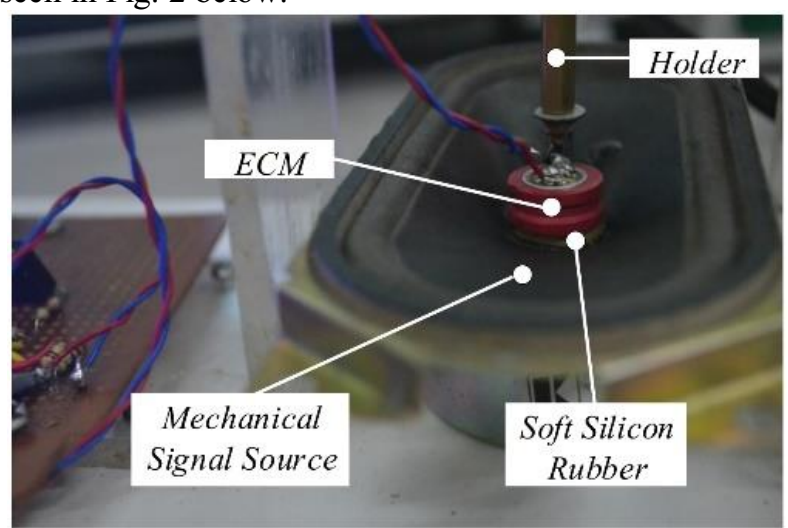

Fig. 2. Signal measurement from Mechanical signal source.

The test results are represented by the change of amplitude of the ECM's response (Fig. 3). The test was performed with 4 different conditions, i.e., without using medium, using a medium with an applied pressure of 5 grams, 40 grams and 400 grams.

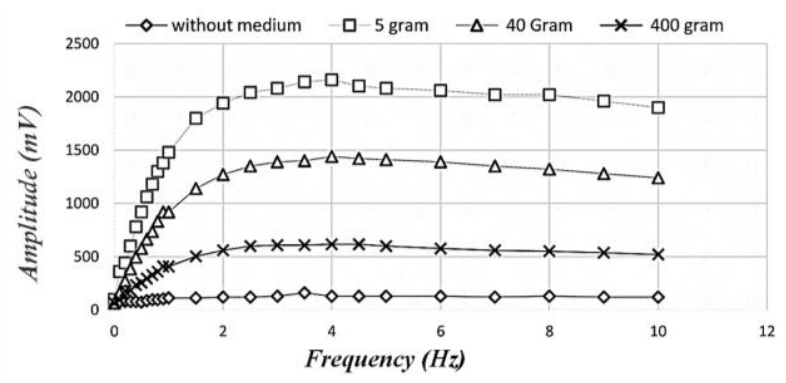

Fig. 3. Amplitude response under various frequency.

From Fig. 3 it can be seen that the microphone has a satisfactorily good response in the frequency range 2 -10 Hz. The amplitude is larger by applying smaller pressure. The largest response amplitude was obtained with a pressure of 5 grams. The errors of the measurement were then determined and plotted in Fig. 4.

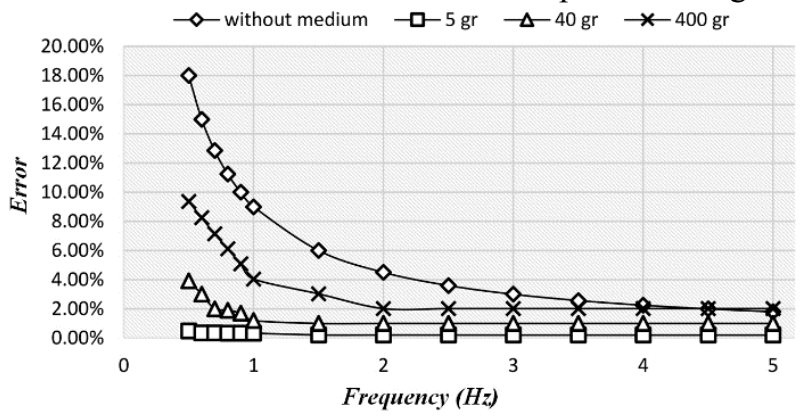

Fig. 4. The error of the measurement under various pressure.
It is obvious that the errors were large at the lower frequency. Furthermore, as can be seen in Fig. 4, the applied pressure affects the errors significantly. The smallest error was found when the response was measured at the smallest pressure i.e., 5 grams. Even at very low frequency the error is still very small. This opens a possibility of constructing a signal processing which can partially amplify the lower frequency part i.e., $0.5-2 \mathrm{~Hz}$ to achieve the goal of using the ECM as the pulse sensor.

\section{Conclusions}

This study proposed a soft silicon rubber as a medium between subjects and ECM. The technique was designed to enhance the mechanical coupling between the microphone and the source signal without affecting the signal source. This ECM can capture signals in the range of $0.5-5 \mathrm{~Hz}$ well without changing shape. ECM also has low complexity and easy to apply and require a low cost. Research in the future can be focused on digital processing to be easily monitored and analyzed based on wireless communication.

The research project was supported by Department of Electrical Engineering, University of Brawijaya

\section{References}

1. H. Kim, J. Y. Kim, Y.-J. Park, and Y.-B. Park, Development of pulse diagnostic devices in Korea Integr. Med. Res., vol. 2, no. 1, pp. 7-17, (2013)

2. C.-Y. C. and C.-Y. LIn, Development of a TCMbased Pulse Impedance Measurement System, in Proceeding o0f the 2017 IEEE/SICE International Symposium on System Integration, (2017)

3. Y. W. Chu, C. H. Luo, Y. F. Chung, C. S. Hu, and C. C. Yeh, Using an array sensor to determine differences in pulse diagnosis-Three positions and nine indicators Eur. J. Integr. Med., vol. 6, no. 5, pp. 516-523, (2014)

4. S. Nomura, Y. Hanasaka, M. Hasegawa-Ohira, T. Ishiguro, and H. Ogawa, Identification of human pulse waveform by silicon microphone chip, Conf. Proc. - IEEE Int. Conf. Syst. Man Cybern., pp. 1145-1150, (2011)

5. E. Yudaningtyas, D. H. Santjojo, W. Djuriatno, I. Siradjuddin, and M. R. Hidayatullah, Identification of Pulse Frequency Spectrum of Chronic Kidney Disease Patients Measured at TCM Points Using FFT Processing, IEEE Explor. 15th Int. Conf. Qual. Res. Int. Symp. Electr. Comput. Eng., pp. 169-172, (2018)

6. E. Yudaningtyas, W. Djuriatno, and R. Yuwono, Pulse frequency spectrum of subjects whose normal electrocardiogram (ECG) test, ARPN J. Eng. Appl. Sci., vol. 10, no. 16, pp. 6859-6862, (2015)

7. P. Wang, W. Zuo, H. Zhang, and D. Zhang, Design and implementation of a multi-channel pulse signal 
acquisition system, 2012 5th Int. Conf. Biomed. Eng. Informatics, BMEI 2012, no. Bmei, pp. 10631067, (2012)

8. T. Lee, K. Shin, and S. Yoo, A case control study to evaluate abnormal lipid profiles in elderly Koreans using radial artery pulse signals, Eur. J. Integr. Med., vol. 5, no. 4, pp. 332-338, (2013)

9. C. S. Hu, Y. F. Chung, C. C. Yeh, and C. H. Luo, Temporal and Spatial Properties of Arterial Pulsation Measurement Using Pressure Sensor Array, Evidence-Based Complement. Altern. Med., vol. 2012 pp. 1-9, (2012)

10. Y. F. Chung, C. S. Hu, C. C. Yeh, and C. H. Luo, How to standardize the pulse-taking method of traditional Chinese medicine pulse diagnosis, Comput. Biol. Med., vol. 43, no. 4, pp. 342-349, (2013)

11. V. Gibbs, D. Cole, and A. Sassano, Ultrasound Physics and Technology, 1st Editio. (Churchil Livingstone Elsevier, 2009)

12. D. Ensminger and L. J. Bond, Ultrasonics: Fundamentals, Technologies, and Applications, 3rd Editio. (Taylor \& Francis Group, 2011)

13. N. S. Inc, Acoustic Properties of CommonMaterials, [Online]. Available: http://www.ndtsystems.com/Reference/Velocity_T able/velocity_table.htm. [Accessed: 12-Apr-2018]. (2013)

14. CUI Inc, Cma-4544Pf-W Description: Electret Condenser Microphone. p. 4, (2013) 